\title{
Cascaded Optical Communication Systems with In-Line Semiconductor Optical Amplifiers
}

\author{
Marina Settembre, Francesco Matera, Volker Hägele, Ildar Gabitov, Arnold W. Mattheus, \\ and Sergei K. Turitsyn
}

\begin{abstract}
The optimization of the performance of installed standard-monomode fibers based optical transmission systems with in-line semiconductor optical amplifiers (SOA's) is reported in this paper. Both solitons and nonreturn-to-zero (NRZ) signals are studied and their capacities are compared. This investigation is based on numerical simulations by solving the nonlinear Schrödinger equations with the split-step method while the SOA is simulated with two different models that we show to be in a good agreement. Transmissions over distances of the order of several hundreds of kilometers are shown to be possible, and very long distances can be reached expecially if in-line sliding filters are used.
\end{abstract}

Index Terms-ASE, GVD, IM-DD, Kerr, NRZ, SOA, solitons, semiconductor.

\section{INTRODUCTION}

$\mathbf{R}$ ECENT impressive progress in the development of optical amplifier technology has stimulated investigations on the next generation of optical transmission systems design as well as studies of different ways to upgrade existing networks. Replacement of electro-optical regenerators by optical amplifiers in presently installed European fiber networks will allow a substantial increase in transmission capacity of the networks. In the third optical window, around $1.55 \mu \mathrm{m}$ wavelength, erbium-doped fibers are considered for the upgrading of existing links, even if in this window the step-index fibers (standard monomode fiber SMF), which constitute the majority of the installed fibers in Europe, show a high-chromatic dispersion or group velocity dispersion (GVD).

Another promising way to upgrade installed lines is to exploit the $1.3 \mu \mathrm{m}$ optical window, where the step-index fibers have their zero dispersion wavelength, using wide-bandwidth polarization-insensitive SOA's, that have been developed recently [1]. This method takes advantage of both the low dispersion of the SMF at this carrier wavelength and attractive features of semiconductor optical amplifiers (SOA's). The

Manuscript received November 12, 1996; revised March 19, 1997. The work was supported in the framework of the ACTS Project UPGRADE.

M. Settembre and F. Matera are with Fondazione Ugo Bordoni, 00142 Rome, Italy.

V. Hägele is with the Universität Kaiserslautern, D-67653 Kaiserslautern, Germany.

I. Gabitov is with the Landau-Institute for Theoretical Physics, Moscow V 334, Russia.

A. W. Mattheus is with Deutsche Telekom AG, Technologiezentrum, D64295 Darmstadt, Germany.

S. K. Turitsyn is with the Institut fur Theoretische Physik I, Heinrich-HeineUniversität Dusseldorf, 40225 Dusseldorf 1, Germany.

Publisher Item Identifier S 0733-8724(97)04350-8.
SOA's cover a broad wavelength range from 0.7 to $1.6 \mu \mathrm{m}$, they are pumped electrically and are substantially cheaper than fiber amplifiers, therefore, the SOA is the natural candidate for incorporation in all-optical transparent transmission systems. There are two major negative factors in the utilization of SOA's in the communication systems: saturation effects, which lead to nonequal amplification of pulses in the pattern in the case of high-bit-rate transmission and additional chirp that a pulse acquires after passing an amplifier. Recent studies have demonstrated the feasibility of $10 \mathrm{~Gb} / \mathrm{s}$ transmission over a $500 \mathrm{~km}$ optical link consisting of SMF and in-line SOA's [1]-[2].

In this work we present an investigation on the use of inline SOA's to obtain high capacity transmission in optical links based on standard step-index fibers at $1.3 \mu \mathrm{m}$. We study here the optimization of the performance for intensity modulated direct detection (IM-DD) systems. Optimization of the system performance is important for practical realization of upgrading of existing networks. From the viewpoint of practical applications we focus on searching for regimes and system parameters for which transmission capacity is maximal assuming other conditions are fixed. Transmission in returnto-zero (RZ) and nonreturn-to-zero (NRZ) format is compared [3]. We study also limitations on the transmission distance and bit rate for both cases: a basic system containing fibers alternating with SOA's as well as an upgraded system with sliding filters [2], [4].

In order to evaluate the system performance, we have simulated the $Q$-factor [3], [5] as a function of operating wavelength and input pulse power.

\section{MODEL}

In the adopted numerical model the fiber propagation is evaluated by solving the coupled nonlinear Schrödinger equations [3] including the effects of the fiber loss, of the GVD and of the Kerr nonlinearity.

The SOA is modeled by the rate equations [6] including noise influence [7]. The rate equation for the electric field envelope $\mathrm{A}$ in the amplifier is

$$
\frac{\partial A}{\partial z}+\frac{1}{V_{g}} \frac{\partial A}{\partial t}=\frac{1}{2}\left(1-i \alpha_{H}\right) g(N) A-\frac{1}{2} \alpha_{\mathrm{int}} A+\frac{1}{V_{g}} A_{s p}
$$

where $V_{g}$ is a group velocity in SOA, $N$ is the carrier density, the carrier-induced index change is accounted for through the 
linewidth enhancement factor $\alpha_{H}$, introduced by Henry [6]. Its typical value for semiconductor lasers and optical amplifiers are in the range $3 \leq \alpha_{H} \leq 8 . g$ is the local power gain defined as $g(N)=\Gamma a\left(N-N_{0}\right)$, where $\Gamma$ is the confinement factor, $N_{0}$ is the carrier density required for transparency and $a$ is the gain coefficient. The rate equation of $g$ is

$$
\frac{\partial g(N)}{\partial t}=\frac{g_{0}-g(N)}{\tau_{c}}-\frac{g(N)|A|^{2}}{E_{\mathrm{sat}}}
$$

where the small-signal gain $g_{0}$ is related to the unsaturated single-pass amplifier gain $G_{0}$ by the ideal gain that should compensate for the losses, that is $G_{0}=\exp \left(\alpha L_{\mathrm{amp}}\right)$, being $\alpha$ the fiber loss and $L_{\text {amp }}$ the amplifier spacing. $\tau_{c}$ is the carrier life time, the saturation energy $E_{\text {sat }}$ determines the pulse energy above which the amplifier is heavily saturated. Typically $E_{\mathrm{sat}}=5-10 \mathrm{pJ}$ for index-guided semiconductor amplifiers [6]. The random spontaneous emission process $A_{\mathrm{sp}}$ is modeled (on the time scales that are interest for this problem) by a complex Gaussian white noise term with zero mean value. It was assumed that the temporal properties of the noise in each strip of the SOA are not affected by photons of the subsequented or previous stripes. The auto-correlation function of the noise process is described by

$$
\left\langle A_{\mathrm{sp}}(t) A_{\mathrm{sp}}^{*}\left(t^{\prime}\right)\right\rangle=\beta_{\mathrm{sp}} P_{\mathrm{sp}}(N) \delta\left(z-z^{\prime}\right) \delta\left(t-t^{\prime}\right) / \tau_{c}
$$

where $\beta_{\mathrm{sp}}$ is the fraction of the total generated spontaneous emission power $P_{\mathrm{sp}}(N)$ coupled into the guided signal mode.

The solution of the rate equations requires a long computation time. This is an important limiting factor in the numerical evaluation of the system performance in this approach. Therefore, we have developed an alternative method for simulations of SOA including noise influence.

By using the approximation in which the internal loss is smaller than the gain, a simplified model for SOA can be adopted that is based on the solution of a simpler equation for the gain $G$ given by [7]

$$
\frac{d G}{d t}=\frac{G_{0}-G}{\tau_{c}}-\frac{P_{\mathrm{in}}(t)}{E_{\mathrm{sat}}}\left[e^{G}-1\right]
$$

where $P_{\text {in }}=|A(z=0)|^{2}$ is the signal power at the SOA input, $P_{\text {sat }}=E_{\text {sat }} / \tau_{c}$ is the saturation power. The signal phase is modulated by the gain saturation according to the equation

$$
\phi_{\text {out }}(t)=\phi_{\text {in }}(t)-\frac{1}{2} \alpha_{H} G(t)
$$

where $\phi_{\text {in }}$ and $\phi_{\text {out }}$ are the signal phases at the input and output of the SOA respectively. Since the split-step method works with periodical signals in time, (4) must be solved with the boundary condition that the gain has to assume the same value in the first and the last point of the time window.

The effect of the amplified spontaneous emission (ASE) is taken into account by adding to each temporal polarized component of the field independent Gaussian noise terms with an energy $n_{\mathrm{sp}} h \nu\left[(G(t)-1]\right.$, where $n_{\mathrm{sp}}$ is the spontaneous emission factor, $h$ the Plank constant and $\nu$ the carrier frequency [8]. The approximation used for the ASE generation is valid provided a high saturation level is not reached and this condition is always satisfied in the cases treated in this paper. Even though the SOA is characterized by a very wide optical bandwidth, the behavior of the signal depends on a narrower bandwidth that is the one due to the receiver and to the bandwidth of nonlinear interaction between signal and ASE. In the case of soliton propagation the bandwidth of nonlinear interaction between signal and ASE is of the order of the signal bandwidth. The effect of the saturation of the optical amplifiers by the ASE noise is inhibited by the use of in-line optical filters with a bandwidth of $640 \mathrm{GHz}$. With a good approximation, assuming a constant GVD along the link, the bandwidth in which the nonlinear interaction between signal and ASE manifests is of the order of $\left[\beta_{2} L_{\mathrm{amp}}\right]^{-1 / 2}$ where $\beta_{2}$ is the GVD [9].

The results of the two models have been compared and in Section III, we will show the good agreement, assuming the condition to generate the same ASE power at the SOA output.

The transmitted data stream consists of a sequence of 32 bits and the pulses are shaped as an hyperbolic secant with a pulse width full width at half maximum (FWHM) of $\tau_{\text {FWHM }}=1.763 \tau_{s}=1 /(5 R)$ (where $R$ is the signal bit rate) in the case of solitons and as a raised cosine function with a rise time equal to $1 /(4 R)$ in the case of NRZ signals.

At the receiver the signal is filtered by an optical Fabry-Perot filter whose bandwidth is $4 R$. The signal is detected with a direct detection receiver (IM-DD system) that consists of a pin photodiode located after the filter and the output electrical signal is filtered by a second order Buttherworth filter with a bandwidth equal to $0.8 R$. The system performance is evaluated in terms of the $Q$ factor, taking into account the patterning effects [3], [5]. The $Q$ factor was obtained on the basis of 25 simulative runs that differ in the ASE generation and in the random rotation of the fiber birefringence axis. We remember that in the hypothesis of Gaussian distribution of the decision variable $Q>6$ corresponds to an error probability lower than $10^{-9}$ [3]. In the study of the system performance, we have also evaluated the timing jitter taking into account the patterning effect. However, in the considered systems, we have observed that, when the $Q$ factor assumed a value higher than six, the standard deviation of the time jitter assumed a value smaller than $6 \%$ of the bit time. In this condition the effect of the time jitter can be neglected [10].

The parameters common in the simulations are: the amplifier spacing $\left(L_{\mathrm{amp}}\right)$ is $50 \mathrm{~km}$, the fiber loss $(\alpha) 0.4 \mathrm{~dB} / \mathrm{km}$, the third order chromatic dispersion $0.1 \mathrm{ps}^{3} / \mathrm{km}$ and the Kerr nonlinearity $(\gamma)$ equal to $2 W^{-1} \mathrm{~km}^{-1}$. The spontaneous emission factor $\left(n_{\mathrm{sp}}\right) 5, \alpha_{H}=5$, the saturation power 30 $\mathrm{mW}, \tau_{c}=200 \mathrm{ps}$ [1] and the semiconductor loss coefficient, containing the internal and mirror losses, $20 \mathrm{~cm}^{-1}$. Effects of polarization mode dispersion and polarization dependent losses can be neglected for the distances studied in this paper [9].

\section{RESULTS}

In Fig. 1 a comparison among the results obtained with the two models is reported. The results refer to the soliton propagation in a link $500 \mathrm{~km}$ long with a GVD $\beta_{2}=$ $-1.5 \mathrm{ps}^{2} / \mathrm{km}$ and in Fig. 1 we report the output signal in terms 


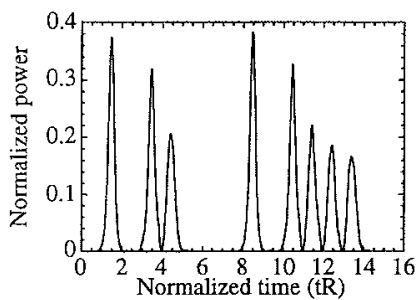

(a)

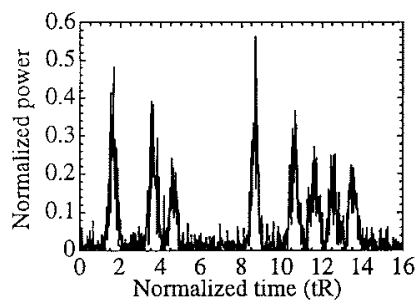

(b)

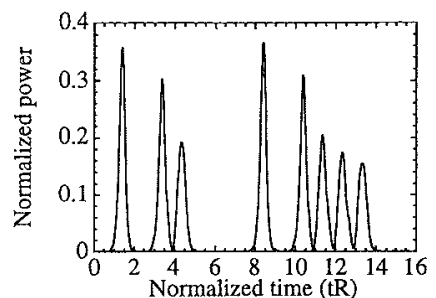

(c)

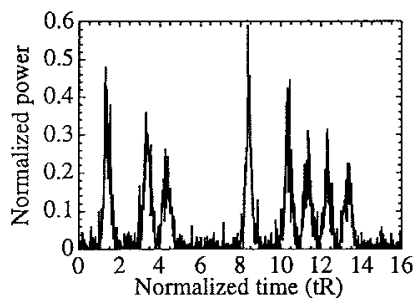

(d)
Fig. 1. Soliton signal at the output of a link $500 \mathrm{~km}$ long with a GVD $\beta_{2}=-1.5 \mathrm{ps}^{2} / \mathrm{km}$. The power is normalized to the peak power of the fundamental soliton and the time to the bit time $1 / R$. Cases (a) and (b) were obtained by solving the rate equations (1), while (c) and (d) by solving (4); (a) and (c) are wihout ASE, while (b) and (d) are with ASE. The time distance among the pulses is $100 \mathrm{ps}$ and $\tau_{s}=11.34 \mathrm{ps}$ corresponding to a bit rate of $10 \mathrm{~Gb} / \mathrm{s}$ and the input peak power of $27.1 \mathrm{~mW}$.

on the normalized power $P / P_{k}$, where $P_{k}$ is the nominal peak power of the fundamental soliton in presence of periodical amplification, given by

$$
P_{k}=\frac{\alpha L_{\mathrm{amp}} G \beta_{2}}{(G-1) \gamma \tau_{s}^{2}}
$$

versus the normalized time $(t R)$, being $R$ the signal bit rate. Only the sequence of 16 bits is reported.

Cases (a) and (b) were obtained by solving (1) and (2), while (c) and (d) by solving (4); (a) and (c) are wihout ASE, while (b) and (d) with ASE. The time distance among the pulses is $100 \mathrm{ps}$ and $\tau_{s}=11.34 \mathrm{ps}$ corresponding to a bit rate of 10 $\mathrm{Gb} / \mathrm{s}$ and the input peak power of $27.1 \mathrm{~mW}$.

As shown by the figure the agreement among the results of the two methods is quite good. We have also compared the two models in terms of $Q$ factor and we have satisfied that considering different propagation conditions the maximum difference given by the two models is smaller than $20 \%$. Due to this reason in the following we only present results by solving (4) since the computer calculation is faster.

In Fig. 2 the $Q$ factor versus the link GVD, $\beta_{2}$, is reported for $10 \mathrm{~Gb} / \mathrm{s} \mathrm{IM-DD} \mathrm{systems} \mathrm{operating} \mathrm{in} \mathrm{a} \mathrm{link} \mathrm{of} 500 \mathrm{~km}$ length with the SOA amplifiers spaced $50 \mathrm{~km}$ apart. Curve (a) refers to soliton signal with $\tau_{s}=11.34$ ps and the input peak power $P$ was chosen equal to $P_{k}$, curve (b) to soliton signal with $P=4 \mathrm{~mW}$ and (c) NRZ signals with $P=4 \mathrm{~mW}$. In case (a), the $Q$ factor decreases lowering the value of $\left|\beta_{2}\right|$ for $\left|\beta_{2}\right|<1 \mathrm{ps}^{2} / \mathrm{km}$, and it is due to the expression of $P_{k}$ that is a linear function of the GVD. For $\left|\beta_{2}\right|$ tending to zero the signal to noise ratio tends to zero and as a consequence also the $Q$ factor tends to zero. For this reason, soliton case is also reported as case (b) in which the peak power was kept constant at $4 \mathrm{~mW}$. As can be seen in curve (a) the $Q$ factor decreases

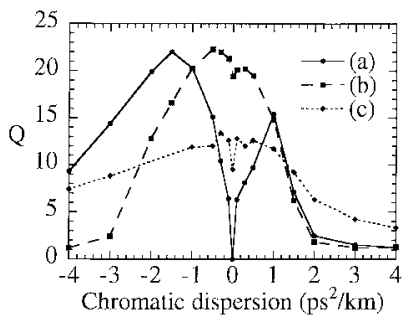

Fig. 2. $Q$ versus input GVD for $10 \mathrm{~Gb} / \mathrm{s}$ IM-DD systems operating in a link $500 \mathrm{~km}$ long. (a) Soliton signal with $P$ equal to nominal peak power of the fundamental solitons. (b) Soliton signal with $P=4 \mathrm{~mW}$ and (c) NRZ signals with $P=4 \mathrm{~mW}$.

in the normal dispersion regime for $\beta_{2}>1 \mathrm{ps}^{2} / \mathrm{km}$ and it is due to the fact that the Kerr nonlinearity does not compensate the GVD degradation that for pulses of $11.34 \mathrm{ps}$ becomes a strong limiting effect. Conversely in the anomalous region soliton signals show a good performance that is maximum for $\beta_{2}=-1.5 \mathrm{ps}^{2} / \mathrm{km}$. For lower values of the GVD the system performance are degraded by the frequency shift induced by the Henry constant and by the fact that the soliton period, $L_{s}=(\pi / 2)\left(\tau_{s}^{2} / \beta_{2}\right)$, tends to become comparable with the amplifier spacing. The latter condition induces soliton instability effects [3], [11]. Curve (b) shows that if soliton pulses are launched with an input power of $4 \mathrm{~mW}$ a high value of the $Q$ factor is reached for $\left|\beta_{2}\right|<1 \mathrm{ps}^{2} / \mathrm{km}$ and it is due to the small degradation induced by the GVD. The smaller value of the $Q$ factor for $\beta_{2}=0$ is due to the strong interactions in terms of FWM between signal and ASE [3]. For a GVD lower than $-1 \mathrm{ps}^{2} / \mathrm{km}$, the impact of the chromatic dispersion cannot be neglected and since (6) is not verified the $Q$ factor is lower with respect to the case (a).

In case (c), the system performance is very good for $\beta_{2}<1 \mathrm{ps}^{2} / \mathrm{km}$. Also NRZ signals show better performance in the anomalous region, since in this region the Kerr effect tends to compensate the GVD also for NRZ signals [3]. It has to be pointed out that in the case of the NRZ signals used in this work the GVD has a weaker degradation with respect to the soliton signals since the response time of NRZ signals is equal to $25 \mathrm{ps}$. This is the reason why for high values of $\left|\beta_{2}\right|$ NRZ signals show higher values of the $Q$ factor with respect to the case (a) in normal dispersion region and with respect to case (b) in anomalous dispersion region. Conversely, for low values of $\left|\beta_{2}\right|$ case (c) exhibits a lower value of the $Q$ factor with respect to the case (b).

To better explain the effects shown in Fig. 2, in Fig. 3 we report on a comparison between the behavior of NRZ and soliton signals operating in the $500 \mathrm{~km}$ link with a GVD equal to $-0.1 \mathrm{ps}^{2} / \mathrm{km}$; case (a) refers to NRZ signals in absence of ASE, (b) NRZ signals in presence of ASE, (c) is the eye diagram at the output of the electrical filter in the case (b), while cases (d), (e), and (f) are the same cases of the figures (a), (b), and (c) but for soliton signals. The power are normalized to the peak power equal to $4 \mathrm{~mW}$. As shown by the Fig. 3(a), the saturation effect of the optical amplifiers produces a strong reashaping of the NRZ signals cutting part of the power on each bit. Such an effect is much less relevant in the case of the soliton signals (case d) since the time duration of the pulse 


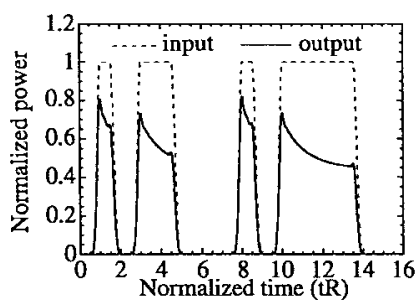

(a)

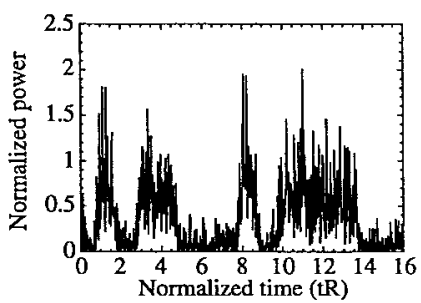

(b)

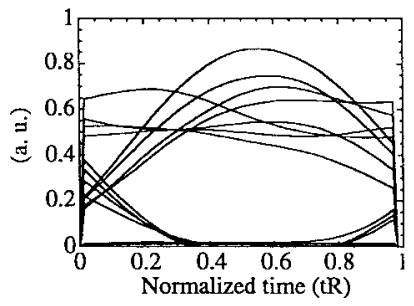

(c)

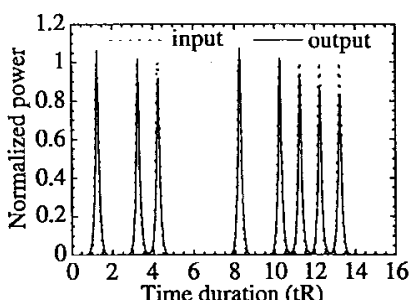

(d)

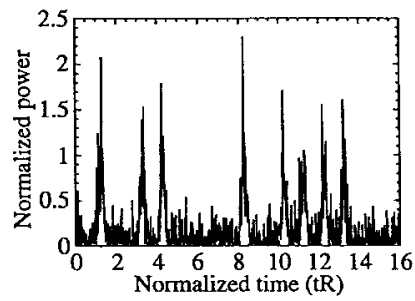

(e)

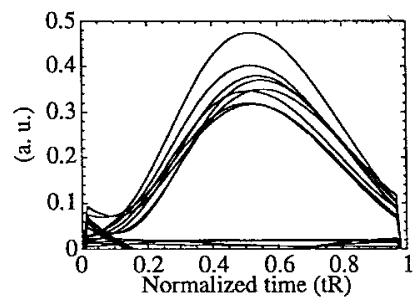

(f)
Fig. 3. Comparison between the behavior of NRZ and soliton signals operating in the link of Fig. 2 with a GVD equal to $-0.1 \mathrm{ps}^{2} / \mathrm{km}$; case (a) refers to NRZ signals in absence of ASE, (b) NRZ signals in the presence of ASE, (c) is the eye diagram at the output of the electrical filter of the case (b), while cases (d), (e), and (f) are the same cases of the figures (a), (b), and (c), respectively, but for soliton signals. The power is normalized to the peak power of $4 \mathrm{~mW}$.

is much shorter than the carrier life time. Due to this fact, in the case of the NRZ signal a stronger reduction of the signal to noise ratio is present, and as a consequence a smaller $Q$ factor can be observed.

It has to be pointed out as the eye diagram show strong fluctuations in the "1" level that could indicate a bad performance of the system. Such a behavior is due to patterning effect and as a consequence the standard deviation of the noise remains low, and adopting the definition of the $Q$ factor as defined in [5], the system shows high values of the $Q$ factor as shown by the Fig. 2 .

In Fig. 4 the $Q$ factor versus input peak power is reported for $10 \mathrm{~Gb} / \mathrm{s}$ soliton transmission over $500 \mathrm{~km}$ in IM-DD systems with the SOA amplifiers spaced $50 \mathrm{~km}$ apart. The values of the GVD are reported in the figure and $\tau_{s}=$ 11.34 ps. For low input power the $Q$ factor assumes low values since the signal-to-noise ratio is low and for a GVD lower than $-2 \mathrm{ps}^{2} / \mathrm{km}$ the signal is strongly degraded by the chromatic dispersion, that is not compensated by the Kerr effect. Increasing the power the $Q$ factor grows both due to the improvement of the signal-to-noise ratio and to the compensation of GVD by means of the Kerr effect.

When the input peak power reaches the value given by (6) the system shows an high value of $Q$ factor since soliton

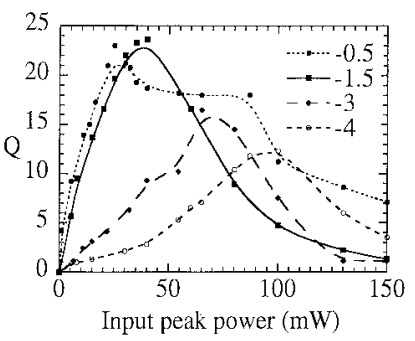

Fig. 4. $Q$ versus input peak power for for $10 \mathrm{~Gb} / \mathrm{s}$ soliton IM-DD systems operating in a link $500 \mathrm{~km}$ long. The values of the GVD are reported in the figure.

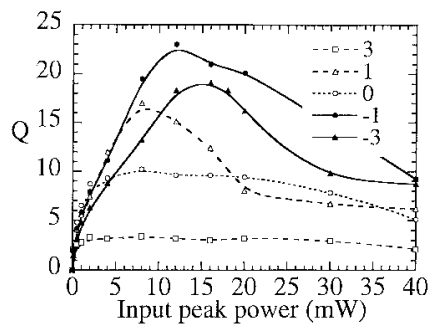

Fig. 5. $Q$ versus input peak power for $10 \mathrm{~Gb} / \mathrm{s}$ NRZ IM-DD systems operating in a link $500 \mathrm{~km}$ long. The values of the GVD are reported in the figure.

behaves similar to the ideal case. In agreement with (6) $P_{k}=9,27.1,54.2$, and $72.26 \mathrm{~mW}$ for the values of GVD equal to $-0.5,-1.5,-3$, and $-4 \mathrm{ps}^{2} / \mathrm{km}$. When the input power is higher than $P_{k}$ several effects can occur. Generally the soliton can show a time narrowing due to the fact that its time duration tends to be equal to the one that can be obtained by the condition $P=P_{k}$. In this condition the soliton can still propagate maintaining its shape, and as a consequence an increase of the $Q$ factor can be achieved due to the presence of a higher power that improves the signal-to-noise ratio (SNR). This behavior is possible until the time duration of the pulse becomes so narrow that, due the presence of soliton instability effect induced by the periodical amplification, soliton breaks up in a stream of pulses and the system performance becomes very poor. It has to be pointed out that the presence of the gain saturation limits the level of the power along the link reducing the effect of the soliton instability.

Soliton propagation shows a good stability, in terms of tolerance of the input power, for $\beta_{2}>-1.5 \mathrm{ps}^{2} / \mathrm{km}$. For lower value the process of the soliton instability results more detrimental.

In Fig. 5 the $Q$ factor versus input peak power is plotted for $10 \mathrm{~Gb} / \mathrm{s}$ NRZ IM-DD systems operating in $500 \mathrm{~km}$ links with the SOA amplifiers spaced $50 \mathrm{~km}$ apart. The values of the GVD are reported in the figure. As in the previous case of the soliton signals, the system shows a growth of the $Q$ factor as a function of the input power until reaching the threshold in which the negative impact of the Kerr nonlinearity shows up. Comparing the same values of GVD we can see how in the anomalous region the performance is better and it is due to the nonlinear compensation of the GVD by means of the Kerr effect [3]. 


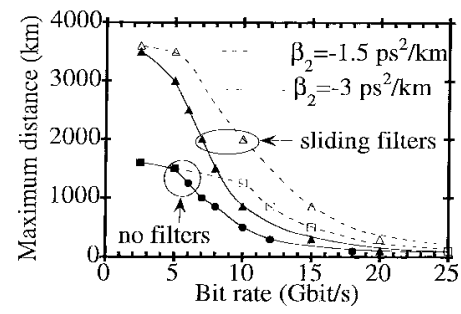

Fig. 6. Transmission distance versus signal bit rate for IM-DD soliton systems operating in a links with the SOA amplifiers spaced $50 \mathrm{~km}$ apart. The values of the GVD are reported in the figure.

The systems show the best performance for an input peak power in the range of $5-15 \mathrm{~mW}$. In the case of $\beta_{2}=0$ the $Q$ factor assumes lower values with respect to the cases in which $\beta_{2}$ is small but different from zero and it is due to the fact that the nonlinear interaction between signal and ASE is deeply reduced in presence of GVD.

Figs. 4 and 5 show how very good transmissions in a link $500 \mathrm{~km}$ long encompassing step-index fibers can be obtained by means of the in-line SOA's both using NRZ and soliton signals even though a weak preference is shown by soliton systems.

As is well known, sliding filters can deeply improve the performance of soliton systems [4]. In a previous work it was found that soliton systems in links with SOA reach the best performance when the strength of the filter, defined as $s=1 /\left(2 \pi^{2} B_{F}^{2} L_{\mathrm{amp}} \beta_{2}\right)$ ( $B_{F}$ is the filter bandwidth) is about 0.1 and sliding parameter $\Delta \phi=2 \pi \tau_{s}^{3} \delta f / L_{\mathrm{amp}} \beta_{2}(\delta f$ is the frequency shift at each filter position) about 0.034 [8].

According to these filter conditions we have evaluated the maximum capacity that can be reached by soliton system considering both the presence and the absence of sliding filters. The criterion used to find the maximum capacity is the one that, fixed the signal bit rate, the $Q$ factor is evaluated as a function of the input power for different link length and the maximum distance is the one that permits to have a $Q>6$ with a tolerance of the input power of $10 \%$. In such a systems the value of the standard deviation of the measured timing jitter was always lower than $6 \%$ of the bit time [10], value that permits to have an error probability lower than $10^{-9}$.

In Fig. 6 it is shown the transmission distance versus signal bit rate for soliton systems. Two values of the GVD equal to -1.5 and $-3 \mathrm{ps}^{2} / \mathrm{km}$ were considered. As already shown in Fig. 2, the system performance is better for $\beta_{2}=$ $-1.5 \mathrm{ps}^{2} / \mathrm{km}$. The benificial effects of the in-line sliding filters are clearly shown. Independently of the GVD the systems show a maximum propagation distance that is equal to 1600 $\mathrm{km}$ in the absence of filters and to $3500 \mathrm{~km}$ in presence of in-line filters. This limitation is mainly due to the high value of the accumulated ASE noise. For short distances the main limitation is given by the instability effect induced by the periodical amplification.

In Fig. 7 it is depicted the transmission distance versus signal bit rate for IM-DD NRZ systems operating in a links with the SOA amplifiers spaced $50 \mathrm{~km}$ apart. The values of the GVD are reported in the figure. The anomalous region permits to achieve an higher capacity, while at the zero dispersion

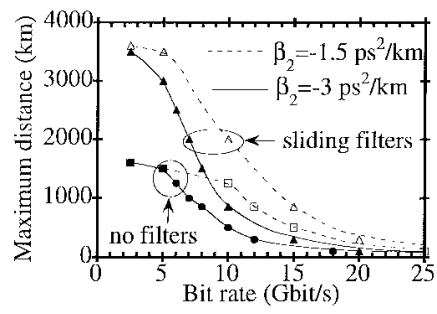

Fig. 7. Transmission distance versus signal bit rate for IM-DD NRZ systems operating in a links with the SOA amplifiers spaced $50 \mathrm{~km}$ apart. The values of the GVD are reported in the figure.

wavelength the nonlinear interaction between signal and ASE induces the strong limitation that does not permit to transmit for distances longer than $900-1000 \mathrm{~km}$.

\section{CONCLUSION}

In this work an investigation on optical systems with in-line SOA has been carried out to show in which conditions the best performance can be achieved. Results show that soliton signals prefer a propagation with a GVD between -1.5 and $-0.5 \mathrm{ps}^{2} / \mathrm{km}$, while in the case of the NRZ signals the GVD should be chosen according the requirement of the system in terms of capacity and distance. For distances shorter than 500 $\mathrm{km}$ a regime with a zero GVD is preferable since it permits the transmission of higher capacity, also with respect to soliton signals. Conversely for distances longer than $500 \mathrm{~km}$ a GVD different from zero is required to limit the nonlinear interaction between signal and ASE. In this case a soliton propagation is preferable, expecially if in-line sliding filters are adopted.

\section{REFERENCES}

[1] C. T. H. F. Liedenbaum, J.J. Reid, L. F. Tiemeijer, A. J. Boot, P. I. Kuindersma, I. Gabitov, and A. Mattheus, "Experimental long haul 1300 $\mathrm{nm}$ soliton transmission on standard single-mode fibers using quantum well laser amplifiers," in Proc. ECOC'94, Florence, Italy 1994, pp. 233-236.

[2] A. Mecozzi, "Soliton transmission control with semiconductor amplifiers," Opt. Lett., vol. 20, pp. 1616-1618, 1995.

[3] F. Matera and M. Settembre, "Comparison of the performance of optically amplified transmission systems," J. Lightwave Technol., vol. 14, pp. 1-11, Jan. 1996.

[4] L. F. Mollenauer, J. P. Gordon, and S. G. Evangelides, "The slidingfrequency guiding filter: an improvement form of soliton jitter control," Opt. Lett., vol. 17, pp. 1575-1577, 1992.

[5] C. J. Anderson and J. A. Lyle, "Technique for evaluating system performance using $Q$ factor in numerical simulations exhibiting inte

[6] rsymbol interference," Electron. Lett., vol. 30, pp. 71-72, 1994.

[7] G. P. Agrawal and N. A. Olsson, "Self-phase modulation in spectral broadening of optical pulses in semiconductor laser amplifiers," IEEE J. Quantum Electron., vol. 25, pp. 2297-2306, 1989.

[8] N. Schunk and K. Peterman, "Noise analysis of injection-locked semiconductor injection lasers" IEEE J. Quantum Electron., vol. 22 pp. 642-650, 1986.

[9] F. Matera and M. Settembre, "Study of the performance of $1.3 \mu \mathrm{m}$ transmission systems on standard step-index fibers with semiconductor optical amplifiers," Opt. Commun., in press.

[10] E. Iannone, F. Matera, A. Mecozzi, and M. Settembre "Performance Evaluations of very long span direct detection intensity and polarization modulated systems," J. Lightwave Technol., vol. 14, pp. 261-272, 1996.

[11] J. V. Wright, S. F. Carter, "Constraints on the design of long-haul soliton systems," in Proc. Nonlinear Guided-Wave Phenomena, Cambridge, England, Sept. 2-4, 1991, pp. 6-10.

[12] L. F. Mollenauer, S. G. Evangelides, and H. A. Haus "Long-distance soliton propagation using lumped amplifiers and dispersion shifted fiber," J. Lightwave Technol., vol. 9, pp. 194-196, 1991. 


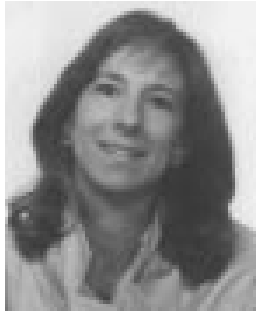

Marina Settembre was born in Rome, Italy, on July 6, 1960. She received the Laurea degree in physics from the University "La Sapienza" of Rome, Italy, in 1985 .

In 1985, she was granted a fellowship at the Fondazione Ugo Bordoni, Rome, Italy, on developments of materials for application in nonlinear optics. Since 1986, she has been a Researcher at the Fondazione Ugo Bordoni working on optical devices for signal routing and processing on numerical modeling of pulse propagation along active and passive fiber links, and on all-optical local and metropolitan area networks.

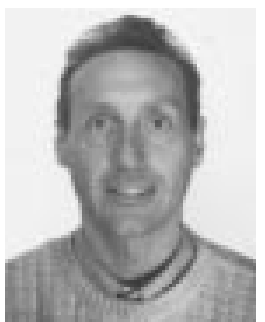

Francesco Matera was born in Rome, Italy, on May 1, 1961. He received the Laurea degree in electronics engineering from the University "La Sapienza" in Rome, Italy, in 1985.

In 1986 he was granted a fellowship at Fondazione Ugo Bordoni, Rome, Italy, on optical fibers. Since 1988, he has been a Researcher at Fondazione Ugo Bordoni, where he works on optical fibers and optical local area networks with the optical Communication Group.

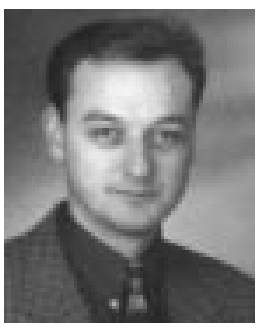

Volker Hägele was born in Stuttgart, Germany, in 1966. He received the Dipl.-Ing. degree from the Department of Electrical Engineering at the University of Kaiserslautern, Kaiserslautern, Germany, in 1993. He received the Dr.-Ing. degree with the Optical Telecommunication Group of the Department of Electrical Engineering at the University of Kaiserslautern in 1996.

His area of research has been in systems theory of optical systems and telecommunications. In 1994, he joined the Department of Mathematical Sciences of the Rensselaer Polytechnic Institute, Troy, NY. Since 1997, he has been working in the HFC Communications Group of the Access System Division of Alcatel SEL in Stuttgart, Germany.
Ildar Gabitov, photograph and biography not available at the time of publication.

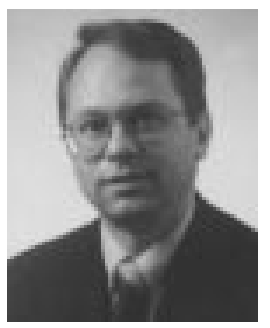

Arnold W. Mattheus received the Ph.D. degree from the University of Kaiserslautern, Kaiserslautern, Germany, in 1987 in the field of laser spectroscopy and molecular collision dynamics.

Afterwards, he joined the Research Centre of the Deutsche Telekom, where he studied coherent optical transmission systems until 1990. He then investigated various techniques for high-capacity data transmission on terrestrial standard monomode fiber-based networks. This work included soliton propagation and passive dispersion compensation. In this context, he has been involved in several European research projects including RACE-TRAVEL, COST 239, and ACTS-UPGRADE. Recently, he extended his research interests to conceptional designs of networks for both long distance traffic and the access layer.

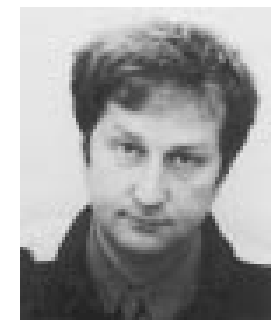

Sergei K. Turitsyn was born on July 17, 1960, in Angarsk, Russia. He received the M.Sc. degree from the Novosibirsk State University, Novosibirsk, Russia, in 1982 and the Ph.D. degree from the Institute of Nuclear Physics, Novosibirsk, Russia, in 1986.

In 1982, he joined the Institute of Automatics and Electrometry, Siberian Branch of the Russian Academy of Science, Novosibirsk. His research was in the fields of nonlinear waves and soliton dynamics with applications to nonlinear optics, plasma physics, and hydrodynamics; and soliton stability; theory of self-focusing and collapse. Since 1992, he has been a Visiting Researcher in the institute of Theoretical Physics I, University of Dusseldorf, Dusseldorf, Germany. His current activities are in the fields of optical fiber transmission, soliton theory, stability of nonlinear waves, self-focusing and wave collapse, and dynamics of the coherent structures in lattices. 